\title{
UPPER BOUNDS FOR A BLOCH CONSTANT
}

POU-SHUN CHIANG AND A. J. MACINTYRE

1. Introduction. It seems that the first numerical results concerning Bloch's theorem for an annulus arise from two examples discussed by Valiron [1]. He used a slightly different constant from the one we consider. It is, however, easy to modify his construction. In our notation Valiron's methods lead to the inequality $B(2)<0.10025$. We give a very simple example to show that $B(2)<0.0884$, and another depending on elliptic functions to show that $B(2)<0.0746$.

Definition. Let $w=f(z)=z+a_{2} z^{2}+\cdots$ be regular in $|z|<1$, then we define $B(2)$ as the supremum of all numbers $\lambda$ such that the Riemann surface of the inverse function $z=f^{-1}(w)$ contains a schlicht annulus of the form $\rho<|w|<2 \rho+\epsilon$ cut along a radius where $\epsilon>0$, and $\rho \geqq \lambda$. For the existence of $B(2)$ see [2].

Valiron's first example which was due to H. Cartan is as follows:

Consider the function

(1) $w=f(z)=\frac{U(z)-U(0)}{U^{\prime}(0)}, \quad U(z)=\frac{Z}{(Z-1)^{2}}, \quad Z=e^{(z-1) /(z+1)}$.

By elementary calculations, we find

$$
B(2)<0.1156 \text {. }
$$

The second example is as follows:

Consider the function

(3) $w=f(z)=\frac{U(z)-U(0)}{U^{\prime}(0)}, \quad U(z)=\frac{Z^{a}}{\left(Z^{a}-1\right)^{2}}, \quad Z=e^{(z-1) /(z+1)}$

for $|z|<1$ where $a$ is a positive number.

In this example, $f(z)$ will omit the value

$$
\frac{-U(0)}{U^{\prime}(0)}=\frac{-\left(e^{a}-1\right)}{2 a\left(e^{a}+1\right)},
$$

and all points of the negative axis from

$$
\frac{-1 / 4-U(0)}{U^{\prime}(0)}=\frac{-\left(e^{a}+1\right)\left(e^{a}-1\right)}{8 a e^{a}}
$$

to minus infinity.

Received by the editors February 15, 1965. 
To obtain the best upper bound of $B(2)$ from this example, we must choose $a$ to satisfy

$$
e^{a}=3+\sqrt{ } 8
$$

that is

$$
a \fallingdotseq 1.7627
$$

and have

$$
B(2)<0.10025 \text {. }
$$

(We have deviated from Valiron only in our choice of $a$.)

These two examples of course also limit the constants obtained when the condition "schlicht" is dropped.

2. New examples. The following two examples will give us better estimates for $B(2)$.

EXAMPLE 1. Let us construct the function $w=f(z)=z+a_{2} z^{2}+\cdots$ which is regular in $|z|<1$ and maps $|z|<1$ on the Riemann surface with a winding point of order 2 at $w=\alpha^{*}$ and a cut from $w=2 \alpha^{*}$ radially to infinity.

Consider the function $w$ of $z$ defined by

(1) $\quad V=\frac{z+i \alpha}{1-i \alpha z}, \quad U=V^{2}, \quad W=\frac{U}{(1+U)^{2}}, \quad w=c\left(W+\frac{1}{4}\right)$.

Note that $|z|<1$ is represented in this way on a Riemann surface of two sheets over the $W$ plane which has $W=0$ as a winding point and the segments of the positive real axis from $1 / 4$ to infinity as cuts. The corresponding positions of winding point and end of cut in the $W+1 / 4$ plane will be $1 / 4$ and $1 / 2$. We also require $z=0$ to correspond to $w=0$.

Let us set $W=-1 / 4$ for $U=-\alpha^{2}$, then $U=-3 \pm \sqrt{ } 8$ and $V$ $= \pm i \alpha$. Hence

$$
\alpha=\sqrt{ } 2-1 .
$$

It only remains to find the value of $c$ such that $f^{\prime}(0)=1$, and then $\alpha^{*}$ will equal $c / 4$. For this, since

$$
\begin{aligned}
\frac{d w}{d z} & =c \cdot \frac{1-U}{(1+U)^{3}} \cdot 2 V \cdot \frac{1-\alpha^{2}}{(1-i \alpha z)^{2}}=c \cdot \frac{1-V^{2}}{\left(1+V^{2}\right)^{3}} \cdot 2 V \cdot \frac{1-\alpha^{2}}{(1-i \alpha z)^{2}} \\
\left.\frac{d w}{d z}\right|_{z=0} & =1 \text { implies } c=\frac{\left(1-\alpha^{2}\right)^{2}}{2 i \alpha\left(1+\alpha^{2}\right)}=-\frac{i}{\sqrt{ } 2} .
\end{aligned}
$$


Thus the desired function is as follows;

$$
\begin{aligned}
w & =-\frac{i}{\sqrt{ } 2}\left(W+\frac{1}{4}\right), & W & =\frac{U}{(1+U)^{2}}, \\
U & =V^{2}, & V & =\frac{z+i(\sqrt{ } 2-1)}{1-i(\sqrt{ } 2-1) z} .
\end{aligned}
$$

Since $|c| / 4=\sqrt{ } 2 / 8<0.1768$, we have

$$
B(2)<0.0884 \text {. }
$$

Example 2. This example is in fact an extension of Example 1. Let us construct the function $w=f(z)=z+a_{2} z^{2}+\cdots$ which is regular in $|z|<1$ and maps $|z|<1$ on the Riemann surface with winding points of order 2 at $w=\alpha$ and at $w=2 \alpha$ and a cut from $w=4 \alpha$ radially to infinity.

By using a linear transformation between $|z|<1$ and $|Z|<1$ to map that point in $|z|<1$ which is the corresponding point of the first double point into the origin of the $Z$-plane, we get the system of equivalent regions, $\cdots S^{3}, S^{2}, S^{1}, S_{0}, S_{1}, S_{2}, \cdots$ in $|Z|<1$ (see Figure) where the upper and the lower indices indicate the corresponding sheets of the Riemann surface of the inverse function $z=f^{-1}(w)$ of the desired function. This system of regions, in fact, has infinitely many members related mutually by a transformation which is the composition of a pair of elliptic transformations,

$$
\zeta^{\prime}=-\zeta
$$

and

$$
\frac{\zeta^{\prime}-i \delta}{1+i \delta \zeta^{\prime}}=-\frac{\zeta-i \delta}{1+i \delta \zeta}
$$

in the following way, for instance,

$$
\begin{aligned}
& S_{0} \stackrel{(6)}{\longrightarrow} S_{1} \stackrel{(7)}{\longrightarrow} S^{1} \stackrel{(6)}{\longrightarrow} S_{2} \stackrel{(7)}{\longrightarrow} S^{3} \longrightarrow \\
& S_{1} \stackrel{(6)}{\longrightarrow} S_{0} \stackrel{(7)}{\longrightarrow} S^{2} \stackrel{(6)}{\longrightarrow} S_{3} \stackrel{(7)}{\longrightarrow} S^{\sigma} \stackrel{(7)}{\longrightarrow} \text { etc. }
\end{aligned}
$$

By simple calculation, the composition (6) ०(7) of (6) and (7) will be a hyperbolic transformation

$$
\frac{\zeta^{*}-i}{\zeta^{*}+i}=\left(\frac{1-\delta}{1+\delta}\right)^{2} \cdot \frac{\zeta-i}{\zeta+i}
$$

After this preparation, the desired function will be obtained by the 
successive composition of linear transformations, an exponential and an elliptic function starting from the upper-half plane of a sheet of the Riemann surface.

Let us first find the linear transformation between the upper-half plane of the $w$-plane and the upper-half plane of the $W$-plane such that $w=\alpha, w=2 \alpha, w=4 \alpha, w=\infty$ go to $W=-1 / k, W=-1, W=1$, $W=1 / k$ respectively. The required transformation is

$$
W=\frac{\sqrt{ } 3 w-(3+\sqrt{ } 3) \alpha}{(2 \sqrt{ } 3-3) w-(5 \sqrt{ } 3-9) \alpha}
$$

with $k=2-\sqrt{ } 3$. We also find that

$$
W=W_{0}=-(7+4 \sqrt{ } 3) \stackrel{\bullet}{=}-13.92820,
$$

when $w=0$.

Next we want to find the transformation between the upper-half plane of the $W$-plane and the interior of the rectangle of the $u$-plane such that $W=-1 / k, W=-1, W=1, W=1 / k$ go to $u=-K+i K^{\prime}$, $u=-K, u=K, u=K+i K^{\prime}$ respectively. This is given [3] by

$$
u=\int_{0}^{W} \frac{d W}{\left(\left(1-W^{2}\right)\left(1-k^{2} W^{2}\right)\right)^{1 / 2}}
$$

where

$K=\int_{0}^{1} \frac{d t}{\left(\left(1-t^{2}\right)\left(1-k^{2} t^{2}\right)\right)^{1 / 2}}, \quad K^{\prime}=\int_{0}^{1} \frac{d t}{\left(\left(1-t^{2}\right)\left(1-k^{\prime 2} t^{2}\right)\right)^{1 / 2}}$

and $k^{\prime}=\left(1-k^{2}\right)^{1 / 2}=(4 \sqrt{ } 3-6)^{1 / 2}$. Using tables [4] we have $K \fallingdotseq 1.60047$ and $K^{\prime} \fallingdotseq 2.73955$.

Let $u_{0}$ be the corresponding value of $W_{0}$, then since $s n\left(u_{0}-i K^{\prime}\right)$ $=\left\{k \cdot s n\left(u_{0}\right)\right\}^{-1}$, by using mathematical tables again we have

$$
u_{0} \fallingdotseq-0.27148+2.73955 i \text {. }
$$

After (11) we continue to apply the following successive transformations:

$$
\begin{aligned}
& U=u+K \\
& V=i \pi U / 4 K \\
& \zeta=\exp \left(V+\pi K^{\prime} / 4 K\right)
\end{aligned}
$$




$$
\begin{aligned}
& Z=i \frac{\zeta-1}{\zeta+1} \\
& z=\frac{Z-Z_{0}}{1-Z_{0} Z} .
\end{aligned}
$$

The values $U_{0}, V_{0}, \zeta_{0}$ and $Z_{0}$ which are the values taken at $z=0$ are

$$
\begin{aligned}
& U_{0} \fallingdotseq 1.32899+2.73955 i \\
& \mathrm{~V}_{0} \fallingdotseq-1.34438+0.65217 i \\
& \zeta_{0} \fallingdotseq e^{0.65217 i} \\
& Z_{0} \fallingdotseq-0.33815 .
\end{aligned}
$$

Thus, we are almost in the position to construct the desired function $w=f(z)=z+a_{2} z^{2}+\cdots$. It only remains to find the value $\alpha$ such that $f^{\prime}(0)=1$.

For this, since

$$
\begin{aligned}
\left.\frac{d w}{d z}\right|_{z=0}= & \frac{(4 \sqrt{ } 3-6) \alpha}{\left\{(2-\sqrt{ } 3) W_{0}-1\right\}^{2}} \cdot\left\{-\left(\left(1-W_{0}^{2}\right)\left(1-k^{2} W_{0}^{2}\right)\right)^{1 / 2}\right\} \\
& \cdot \frac{4 K}{\pi i} \cdot \frac{1}{\zeta_{0}} \cdot \frac{2 i}{\left(i-Z_{0}\right)^{2}} \cdot\left(1-Z_{0}^{2}\right)=1 \\
\alpha= & \frac{-\left(i-Z_{0}\right)^{2} \cdot \zeta_{0} \cdot \pi \cdot\left\{(2-\sqrt{ } 3) W_{0}-1\right\}^{2}}{\left(1-Z_{0}^{2}\right) \cdot 8 K \cdot\left(\left(1-W_{0}^{2}\right)\left(1-k^{2} W_{0}^{2}\right)\right)^{1 / 2} \cdot(4 \sqrt{ } 3-6)}
\end{aligned}
$$

Hence by substituting $W_{0}, u_{0}, U_{0}, V_{0}, \zeta_{0}$ and $Z_{0}$ with their numerical values, we have

$$
\alpha<0.1492 \text {. }
$$

Therefore

$$
B(2)<0.0746 .
$$

REMARK. These new examples as well as Valiron's also limit an other kind of Bloch constant, say $B^{*}$, in which the schlicht circle: $\left|w-w_{f}\right|<P$ has its center and a whole diameter on the positive real axis with $P>B^{*}$. To find an upper bound of $B^{*}$, it would be better to set the end of the cut at $w=3 \alpha$ instead of at $w=4 \alpha$ in Example 2 . In this case, the corresponding transformation of (9) will be

$$
W=\frac{-\sqrt{ } 8 w+(4+\sqrt{ } 8) \alpha}{(8-3 \sqrt{ } 8) w+(7 \sqrt{ } 8-20) \alpha}
$$


with $k=3-\sqrt{ } 8$. Hence subsequently $k^{\prime}=(6 \sqrt{ } 8-16)^{1 / 2}, K \fallingdotseq 1.582$ and $K^{\prime} \fallingdotseq 3.169$.

With the other transformations (11), (13), (14), (15), (16), (17) remain unchanged, we can also calculate the values $W_{0}, u_{0}, U_{0}, V_{0}, \zeta_{0}$ and $Z_{0}$, and similarly can find that $\alpha$ is less than 0.16 . Hence

$$
B^{*}<0.08 \text {. }
$$

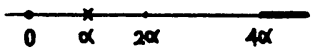

W-PLANE

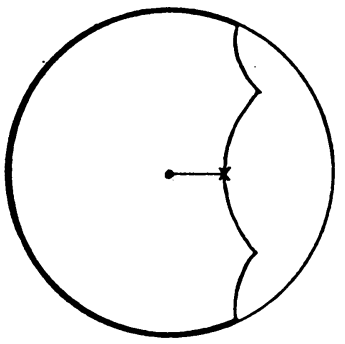

Z-PLANE

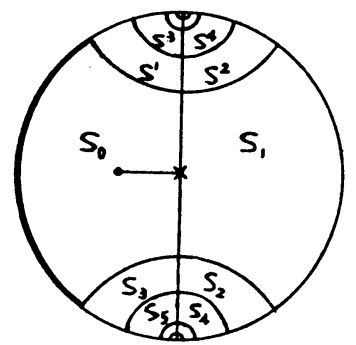

Z-PLANE

\section{REFERENCES}

1. G. Valiron, Sur le théorème de M. Bloch, Rend. Circ. Mat. Palermo 54 (1930).

2. A. J. Macintyre, On Bloch's theorem, Math. Z. 44 (1938), 536-540.

3. H. Kober, Dictionary of conformal representations, Dover, New York, 1952.

4. C. R. C. Standard mathematical tables, 12th ed., Chemical Rubber Publishing Co.

University of Cincinnati AND

Aerospace Research laboratories,

Wright-Patterson Air Force Base, Ohio 